Japan. J. Math.

Vol. 19, No. 2, 1994

\title{
$S$-spaces of Gel'fand-Shilov and differential equations
}

\author{
By Chen Dong and Tadato Matsuzawa
}

(Received February 17, 1992)

\section{Introduction}

In their book, [4], Vol. 2, Chapter IV, Gel'fand and Shilov introduced the spaces of test functions of generalized functions which are called $S$-spaces. In [5], Vol. 3, Chapter II, they used these spaces to investigate the uniqueness of the solutions for the Cauchy problems of partial differential equations.

The main aim of this paper is to characterize the generalized functions of the dual spaces $S^{\prime}$ by using the heat equation and give some application to the problems of differential equations.

In $\S 1$, we shall give the definition of $S$-spaces following [4] and we shall consider the Cauchy problem of the heat equation with initial data in $S$. In $\S 2$, we shall investigate the Cauchy problem of the heat equation with initial data in $S^{\prime}$ in Theorem 2.1. There we get homeomorphic correspondence of the generalized functions of $S^{\prime}$ and the solutions to the heat equation. In $\S 3$, we shall describe the solvability and regularity of solutions to ordinary differential equations with constant coefficients in $S^{\prime}$-spaces by using the heat equation. In $\S 4$, as an example of applications to partial differential equations we shall consider the Laplace equation in $R^{n}(n \geq 2)$ and one dimensional heat equation in $S^{\prime}$-spaces. It turns out that Laplace equation in analytic hypoelliptic in $S^{\prime}$-spaces and the heat equation is not hypoelliptic in $S^{\prime}$-spaces. Further investigation in $S^{\prime}$-spaces will be given in the future.

\section{§1. Spaces $\mathcal{S}_{r}^{s}\left(R^{n}\right)$}

We use general notations such as $|\alpha|=\alpha_{1}+\cdots+\alpha_{n}$ for $\alpha \in Z_{+}^{n}$ and $\alpha$ ! = $\alpha_{1} ! \cdots \alpha_{n}$ !. For $x=\left(x_{1}, \ldots, x_{n}\right) \in R^{n}$ and $\alpha \in Z_{+}^{n}$ we define $x^{\alpha}=x_{1}^{\alpha_{1}} \cdots x_{n}^{\alpha_{n}}$. For $\varphi(x) \in C^{\infty}\left(R^{n}\right)$ and $\beta \in Z_{+}^{n}$ we define

$$
\varphi^{(\beta)}(x)=\left(\partial / \partial x_{1}\right)^{\beta_{1}} \cdots\left(\partial / \partial x_{n}\right)^{\beta_{n}}(\varphi(x)) .
$$

Definition 1.1. The space $\mathcal{S}_{r, A}^{s, B}(r, s \geq 0 ; A, B>0)$ consists of all infinitely differentiable functions $\varphi(x), x \in R^{n}$, satisfying the inequalities

$$
\left|x^{\alpha} \varphi^{(\beta)}(x)\right| \leq C_{\varphi} A^{|\alpha|} B^{|\beta|}|\alpha|^{r|\alpha|}|\beta|^{s|\beta|}, \quad \alpha, \beta \in Z_{+}^{n},
$$


where the constant $C_{\varphi}$ depends on the function $\varphi$. This is a Banach space with the norm

$$
\|\varphi\|=\sup _{\substack{x \in R^{n} \\ \alpha, \beta}} \frac{\left|x^{\alpha} \varphi^{(\beta)}(x)\right|}{A^{|\alpha|} B^{|\beta|}|\alpha|^{r|\alpha|}|\beta|^{s|\beta|}}
$$

The space $\mathcal{S}_{r}^{s}\left(R^{n}\right)$ is defined by the inductive limit

$$
\mathcal{S}_{r}^{s}\left(R^{n}\right)=\underset{A, B}{\lim } \mathcal{S}_{r, A}^{s, B}
$$

REMARK 1.1. Let $\mathcal{S}\left(R^{n}\right)$ be the space of rapidly decreasing functions of L. Schwartz, then by (1.1) we have the inclusion $\mathcal{S}_{r}^{s}\left(R^{n}\right) \subset \mathcal{S}\left(R^{n}\right)(r, s \geq 0)$.

REMARK 1.2 ([4], Vol. 2, Chapter IV). The space $\mathcal{S}_{r}^{s}\left(R^{n}\right)$ is not trivial if one of the following conditions is satisfied:

(i) $\quad r+s \geq 1, r>0, s>0$;

(ii) $\quad r=0, s>1$;

(iii) $r>1, s=0$.

Otherwise it degenerates to the single function $\varphi(x) \equiv 0$.

Let us remark that the Gaussian function $\exp \left[-x^{2}\right]$ belongs to the space $\mathcal{S}_{1 / 2}^{1 / 2}$ (c.f. Theorem 2.1.).

Theorem 1.1 ([4], Vol. 2, Chapter IV). (a) If $r=0$ and $s>1$ we have

$$
\mathcal{S}_{0}^{s}\left(R^{n}\right)=\mathcal{D}^{\{s\}}\left(R^{n}\right),
$$

where $\mathcal{D}^{\{s\}}$ is the space of Gevrey functions of order $s$ with the compact support.

(b) If $r+s \geq 1(r, s>0)$ and $\varphi(x) \in \mathcal{S}_{r}^{s}\left(R^{n}\right)$ we have the inequality

$$
\left|\varphi^{(\beta)}(x)\right| \leq C B^{|\beta|}|\beta|^{s|\beta|} \exp \left[-a|x|^{1 / r}\right], \quad \beta \in Z_{+}^{n},
$$

where the positive constants $C, B$ and a depend on the function $\varphi$.

Theorem 1.2 ([4], Vol. 2, Chapter IV). If $0<s<1$ every function $\varphi(x) \in$ $\mathcal{S}_{r}^{s}\left(R^{n}\right)$ is continued in the entire $z=x+i y$ plane and the following estimate is satisfied:

$$
|\varphi(x+i y)| \leq C \exp \left[-a|x|^{1 / r}+b|y|^{1 /(1-s)}\right], \quad x+i y \in \mathfrak{C}^{n} .
$$

Here the constants $a$ and $b$ are taken such that

$$
a<r /\left(e A^{1 / r}\right), \quad b>\frac{(1-s)}{e}(B e)^{1 /(1-s)} .
$$


THEOREM 1.3. Let $\left\{\varphi_{j}\right\}$ be a sequence in $\mathcal{S}_{r}^{s}\left(R^{n}\right)$.

(a) If $r+s \geq 1(r, s>0)$ then we have

$$
\varphi_{j} \longrightarrow 0 \text { as } j \longrightarrow \infty \text { in } \mathcal{S}_{r}^{s}
$$

if and only if there are positive constants $B$ and a such that

$$
\sup _{\substack{x \in R^{n} \\ \beta}} \frac{\left|\varphi_{j}^{(\beta)}(x)\right|}{B^{|\beta|}|\beta|^{s|\beta|} \exp \left[-a|x|^{1 / r}\right]} \longrightarrow 0 \text { as } j \longrightarrow \infty .
$$

(b) In particular, if $r+s \geq 1$ and $0<s<1$ then

$$
\varphi_{j} \longrightarrow 0 \text { as } j \longrightarrow \infty \text { in } \mathcal{S}_{r}^{s}
$$

if and only if there are positive constants $a$ and $b$ such that

$$
\sup _{x+i y \in \mathfrak{C}^{n}} \frac{\left|\varphi_{j}(x+i y)\right|}{\exp \left[-a|x|^{1 / r}+b|y|^{1(1-s)}\right]} \longrightarrow 0 \text { as } j \longrightarrow \infty .
$$

THEOREM 1.4. Let $u \in\left(\mathcal{S}_{r}^{s}\right)^{\prime}$.

(a) If $r+s \geq 1(0<r, s<\infty)$ then for any pair of positive numbers a and $B$ there exists a positive constant $C$ such that

$$
|u(\varphi)| \leq C \sup _{\substack{x \in R^{n} \\ \beta}} \frac{\left|\varphi^{(\beta)}(x)\right|}{B^{|\beta|}|\beta|^{s|\beta|} \exp \left[-a|x|^{1 / r}\right]} .
$$

(b) In particular if $r+s \geq 1,0<r<\infty$ and $0<s<1$ then for any pair of positive numbers $a$ and $b$ there exists a positive constant $C$ such that

$$
|u(\varphi)| \leq C \sup _{x+i y \in \mathfrak{C}^{n}} \frac{|\varphi(x+i y)|}{\exp \left[-a|x|^{1 / r}+b|y|^{1 /(1-s)}\right]}
$$

The estimates (1.7) and (1.8) are obtained by a standard method so we omit the proofs.

Now we denote by $E(x, t)$ the $n$-dimensional heat kernel:

$$
E(x, t)= \begin{cases}(4 \pi t)^{-n / 2} \exp \left[-x^{2} / 4 t\right], & t>0 \\ 0, & t<0 .\end{cases}
$$

Here we write $x^{2}=x_{1}^{2}+x_{2}^{2}+\cdots+x_{n}^{2}$. 
Theorem 1.5. Let $\varphi(x) \in \mathcal{S}_{r}^{s}\left(R^{n}\right), 1 / 2 \leq r<\infty, 0<s<\infty, r+s \geq 1$. Then we have

$$
U(x, t) \equiv \int_{R^{n}} E(x-y, t) \varphi(y) d y \in \mathcal{S}_{r}^{s}\left(R_{x}^{n}\right), \quad t>0
$$

and

$$
U(x, t) \longrightarrow \varphi(x) \text { in } \mathcal{S}_{r}^{s}\left(R^{n}\right) \text { as } t \longrightarrow 0_{+}
$$

Proof. ' Let $\varphi(x) \in \mathcal{S}_{r}^{s}\left(R^{n}\right), 1 / 2 \leq r<\infty, 0<s<\infty$. By Theorem 1.1 and Theorem 1.3 it is sufficient to prove that for some positive constants $a$ and $B$ it holds that

$$
\sup _{\substack{x \in R^{n} \\ \beta}} \frac{\left|U^{(\beta)}(x, t)-\varphi^{(\beta)}(x)\right|}{B^{|\beta|}|\beta|^{s|\beta|} \exp \left[-a|x|^{1 / r}\right]} \longrightarrow 0 \text { as } t \longrightarrow 0_{+} \cdot
$$

Let $\delta$ be a small positive number. We have

$$
\begin{aligned}
\left|U^{(\beta)}(x, t)-\varphi^{(\beta)}(x)\right|= & \left|\int_{R^{n}} E(x-y, t)\left(\varphi^{(\beta)}(y)-\varphi^{(\beta)}(x)\right) d y\right| \\
= & \left|\int E(y, t)\left(\varphi^{(\beta)}(x-y)-\varphi^{(\beta)}(x)\right) d y\right| \\
\leq & \int_{|y| \leq \delta} E(y, t)\left|\varphi^{(\beta)}(x-y)-\varphi^{(\beta)}(x)\right| d y \\
& +\int_{|y| \geq \delta} E(y, t)\left|\varphi^{(\beta)}(x-y)\right| d y \\
& +\int_{|y| \geq \delta} E(y, t)\left|\varphi^{(\beta)}(x)\right| d y \\
\equiv & I_{1, \beta}+I_{2, \beta}+I_{3, \beta} .
\end{aligned}
$$

We can write symbolically

$$
\begin{aligned}
\left|\varphi^{(\beta)}(x-y)-\varphi^{(\beta)}(x)\right| & \leq\left|\varphi^{(\beta+1)}(x-\theta y)\right||y| \quad(0<\theta<1) \\
& \leq C B^{|\beta|}|\beta|^{s|\beta|} \exp \left[-a|x-\theta y|^{1 / r}\right]|y|
\end{aligned}
$$

then we have

$$
I_{1, \beta} \leq \delta C B^{|\beta|}|\beta|^{s|\beta|} \exp \left[-a / 2|x|^{1 / r}\right]
$$


Next by taking note that $\left|\varphi^{(\beta)}(x-y)\right| \leq C B^{|\beta|}|\beta|^{s|\beta|} \exp \left[-a|x-y|^{1 / r}\right]$ we have

$$
\begin{gathered}
I_{2, \beta} \leq C B^{|\beta|}|\beta|^{s|\beta|}(4 \pi t)^{-n / 2} \int_{|y| \geq \delta} \exp \left[-y^{2} / 4 t-a|x-y|^{1 / r}\right] d y \\
\leq C B^{|\beta|}|\beta|^{s|\beta|} \exp \left[-(a / 4)|x|^{1 / r}\right] \exp \left[-\delta^{2} / 8 t\right] . \\
\int_{|y| \geq \delta} \exp \left[-y^{2} / 8 t+4 a|y|^{1 / r}\right] d y .
\end{gathered}
$$

Using the assumption $1 / r \leq 2$, we have

$$
I_{2, \beta} \leq \delta_{t} C B^{|\beta|}|\beta|^{s|\beta|} \exp \left[-(a / 4)|x|^{1 / r}\right]
$$

where $\delta_{t} \longrightarrow 0$ as $t \longrightarrow 0$. Finally we have

$$
\begin{aligned}
I_{3, \beta} & =\int_{|y| \geq \delta} E(y, t)\left|\varphi^{(\beta)}(x)\right| d y=\left|\varphi^{(\beta)}(x)\right| \int_{|y| \geq \delta} E(y, t) d y \\
& \leq \delta_{t}^{\prime} C B^{|\beta|}|\beta|^{s|\beta|} \exp \left[-a|x|^{1 / r}\right]
\end{aligned}
$$

where $\delta_{t}^{\prime} \longrightarrow 0$ as $t \longrightarrow 0$. Thus we have $U(x, t) \longrightarrow \varphi$ in $\mathcal{S}_{r}^{s}\left(R^{n}\right)$.

\section{§2. Characterization of the spaces $\left(\mathcal{S}_{r}^{s}\right)^{\prime}$}

Proposition 2.1 ([11]). Let $E(x, t)$ be the $n$-dimensional heat kernel. Then $E(\cdot, t)$ is an entire function of order 2 for $t>0$ and we have the following properties on $E$ :

(i) We have

$$
\int_{R^{n}} E(x, t) d x=1, \quad t>0
$$

(ii) There are positive constants $C$ and $a^{\prime}$ such that

$$
\left|E^{|\alpha|}(x, t)\right| \leq C^{|\alpha|+1} t^{-(n+|\alpha|) / 2} \alpha !^{1 / 2} \exp \left[-a^{\prime}|x|^{2} / 4 t\right], \quad t>0,
$$

where $a^{\prime}$ can be taken as close as desired to 1 and $0<a^{\prime}<1$.

TheOREM 2.1. Let $\left(\mathcal{S}_{r}^{s}\right)^{\prime}$ be the strong dual space of $\mathcal{S}_{r}^{s}\left(R^{n}\right)$ and assume $u \in$ $\left(\mathcal{S}_{r}^{s}\right)^{\prime}, 1 / 2 \leq r<\infty$ and $1 / 2<s<\infty$. Then the function $U(x, t)=u_{y}(E(x-y, t))$ is well defined in $R_{+}^{n+1}=\left\{(x, t) ; x \in R^{n}, t>0\right\}$ and satisfies the heat equation:

$$
(\partial / \partial t-\Delta) U(x, t)=0 \text { in } R_{+}^{n+1}
$$

For every $T>0$ and $\varepsilon>0$ there is a positive constant $C$ such that

$$
|U(x, t)| \leq C \exp \left[\varepsilon\left(|x|^{1 / r}+(1 / t)^{1 /(2 s-1)}\right)\right], \quad x \in R^{n}, \quad 0<t<T ;
$$


In the case where $1 / 2 \leq r<\infty$ and $s=1 / 2$ we have the following inequality

$$
|U(x, t)| \leq C_{\varepsilon, t} \exp \left[\varepsilon|x|^{1 / r}\right] \text { in } R_{+}^{n+1}
$$

For any $\varphi \in \mathcal{S}_{r}^{s}\left(R^{n}\right), 1 / 2 \leq r, s<\infty$, we have

$$
\int_{R^{n}} U(x, t) \varphi(x) d x \longrightarrow u(\varphi) \text { as } t \longrightarrow 0 .
$$

Conversely, every $C^{\infty}$-function $U(x, t)$ defined in $R_{+}^{n+1}$ satisfying the conditions (2.3) and (2.4) or $(2.4)^{\prime}$ can be expressed in the form $U(x, t)=u_{y}(E(x-y, t))$ with unique element $u \in\left(\mathcal{S}_{r}^{s}\right)^{\prime}$.

Proof. Let us assume that $u \in \mathcal{S}_{r}^{s}\left(R^{n}\right)^{\prime}, 1 / 2 \leq r, s<\infty$. Then we have obviously $U(x, t) \in C^{\infty}\left(R_{+}^{n+1}\right)$ and (2.3). We shall prove (2.4) and (2.4)' simultaneously. We have by (1.7) and (2.2)

$$
\begin{aligned}
|U(x, t)| & =\left|u_{y}(E(x-y, t))\right| \\
& \leq C^{\prime} \sup _{\substack{y \in R^{n} \\
\beta}} \frac{C^{|\beta|} t^{-n / 2-|\beta| / 2} \beta !^{1 / 2} \exp \left[-a^{\prime}|x-y|^{2} / 4 t\right]}{B^{|\beta|}|\beta|^{s|\beta|} \exp \left[-a|y|^{1 / r}\right]}
\end{aligned}
$$

from where it follows that for any $\varepsilon>0$ if we take $B>0$ sufficiently large and $a>0$ sufficiently small we obtain easily the estimates of type (2.4) in case $s>1 / 2$ and $(2.4)^{\prime}$ in case $s=1 / 2$.

Next we shall show (2.5). For any $\varphi \in \mathcal{S}_{r}^{s}\left(R^{n}\right), 1 / 2 \leq r, s<\infty$, we have by (1.10)

$$
\begin{aligned}
\int_{R^{n}} U(x, t) \varphi(x) d x & =\int u_{y}(E(x-y, t)) \varphi(x) d x \\
& =u_{y}\left(\int E(x-y, t) \varphi(x) d x\right) \longrightarrow u(\varphi) \text { as } t \longrightarrow 0_{+}
\end{aligned}
$$

In order to prove the converse we need the following two lemmas due to Komatsu, [7] and [9].

LEMMA 2.1. Let $s>1$. Then there exist an infinite order differential operator $p(d / d t)=\sum_{0}^{\infty} a_{k}(d / d t)^{k}$ and a function $v(t)$ having the following properties:

(i) For any $\varepsilon>0$ there is a constant $C>0$ such that

$$
\left|a_{k}\right| \leq C \varepsilon^{k} k !^{-s} \quad k=0,1, \ldots
$$

(ii) $\quad \operatorname{supp} v(t) \subset[0,1], \quad v(t) \geq 0$. 
(iii) There exists a function $w(t) \in C_{0}^{\infty}(R)$ such that $\operatorname{supp} w \subset[1 / 2,1]$ and we have

$$
p(d / d t) v(t)=\delta+w(t) \quad \text { in } \quad-\infty<t<\infty
$$

LEMMA 2.2. Let $s>1$ and $f(t)$ be a measurable function in $[0, \infty)$ such that for any $\varepsilon>0$ there exists a constant $C_{\varepsilon}$ satisfying the inequality

$$
|f(t)| \leq C_{\varepsilon} \exp \left[(\varepsilon / t)^{(s-1)}\right] \quad \text { in }[0, \infty)
$$

Then one can find a function $v(t)$ satisfying the conditions in Lemma 2.1 such that we have $f(t) v(t) \in L^{\infty}(0, \infty)$.

Now we are going to prove the converse of Theorem 2.1 dividing it in two cases, (i) case $1 / 2<s<\infty$, (ii) case $s=1 / 2$.

Assume that $1 / 2<s<\infty$ and $U(x, t)$ satisfies (2.3) and (2.4). We take functions $v(t)$ and $w(t)$ satisfying the conditions in Lemma 2.1 and 2.2 with $s$ replaced by $2 s>1$ and define two functions in the half space $R_{+}^{n+1}$ :

$$
g(x, t)=\int_{0}^{\infty} U(x, t+s) v(s) d s
$$

$$
h(x, t)=\int_{0}^{\infty} U(x, t+s) w(s) d s .
$$

We can see that $g(x, t), h(x, t) \in C^{\infty}\left(R_{+}^{n+1}\right)$ and we have

$$
|g(x, t)|,|h(x, t)| \leq C_{\varepsilon} \exp \left[\varepsilon|x|^{1 / r}\right], \quad t>0 .
$$

Moreover $g(x, t)$ and $h(x, t)$ satisfy the heat equation in $R_{+}^{n+1}$ and if we define

$$
g_{0}(x)=\lim _{t \rightarrow 0} g(x, t), \quad h_{0}(x)=\lim _{t \rightarrow 0} h(x, t) \quad \text { in } R^{n}
$$

then we have

$$
g(x, t)=\int E(x-y, t) g_{0}(y) d y=\int_{0}^{\infty} U(x, t+s) v(s) d s
$$

Operating $p(-d / d t)$ for each member of $(2.8)$ we have

$$
\begin{aligned}
U(x, t)+h(x, t) & =p(-d / d t) \int E(x-y, t) g_{0}(y) d y \\
& =\int E(x-y, t) p(-\Delta) g_{0}(y) d y
\end{aligned}
$$


from which we have formally

$$
u \equiv U(x, 0)=p(-\Delta) g_{0}(x)-h_{0}(x) .
$$

We can easily see that $p(-\Delta) g_{0}(x), h_{0}(x) \in\left(\mathcal{S}_{r}^{s}\right)^{\prime}$ since we have the estimates

$$
\left|g_{0}(x)\right|,\left|h_{0}(x)\right| \leq C_{\varepsilon} \exp \left[\varepsilon|x|^{1 / r}\right] \text { in } R^{n}
$$

for any $\varepsilon>0$.

Next we shall prove the case (ii) $s=1 / 2$. We can rewrite the definition of the space $\mathcal{S}_{r}^{1 / 2}, 1 / 2 \leq r<\infty$, by using Theorem 1.4: The space $\mathcal{S}_{r, a}^{1 / 2, B}(a, B>0)$ consists of entire functions $\varphi(z)=\varphi(x+i y)$ such that

$$
\left|\varphi^{(\beta)}(x)\right| \leq C B^{|\beta|} \beta !^{1 / 2} \exp \left[-a|x|^{1 / r}\right], \quad x \in R^{n}, \quad \beta \in Z_{+}^{n}
$$

for some constant $C=C_{\varphi}$. We define

$$
\mathcal{S}_{r}^{1 / 2}=\underset{\substack{a \rightarrow 0 \\ B \rightarrow \infty}}{\lim _{r, a}} \mathcal{S}^{1 / 2, B}
$$

Let $\varphi(x) \in \mathcal{S}_{r, a}^{1 / 2, B}$ and consider the backward Cauchy problem for the heat equation:

$$
\begin{gathered}
(\partial / \partial t-\Delta) u(x, t)=0, \quad 0<t<t_{0} \\
u\left(x, t_{0}\right)=\varphi(x), \quad x \in R^{n}
\end{gathered}
$$

For any pair of positive numbers $a$ and $B$ if we take $t_{0}$ sufficiently small so that $0<t_{0}<1 / 2 B^{2}$ we can find the solution by

$$
u(x, t)=\sum_{k=0}^{\infty} \frac{\left(\left(t-t_{0}\right) \Delta\right)^{k}}{k !} \varphi(x), \quad x \in R^{n} .
$$

We can easily see that $u(\cdot, t) \in \mathcal{S}_{r, a}^{1 / 2, B}, 0 \leq t \leq t_{0}$. Thus we have a continuous linear transformation

$$
\mathcal{S}_{r, a}^{1 / 2, B} \ni \varphi \longrightarrow u(x, 0)=\exp \left[-t_{0} \Delta\right] \varphi \in \mathcal{S}_{r, a}^{1 / 2, B}
$$

Now for $U(x, t)$ satisfying $(2.3)$ and $(2.4)^{\prime}$ we define $u_{0} \in\left(\mathcal{S}_{r, a}^{1 / 2, B}\right)$, by

$$
u_{0}(\varphi)=\int U\left(x, t_{0}\right) \exp \left[-t_{0} \Delta\right] \varphi(x) d x, \quad \varphi \in \mathcal{S}_{r, a}^{1 / 2, B}
$$

Then $u_{0}$ doesn't depend on $t_{0}, 0<t_{0}<1 / 2 B^{2}$, and we get $u_{0} \in\left(\mathcal{S}_{r, a}^{1 / 2, B}\right)^{\prime}$. We shall show that $u_{0}=\lim _{t \rightarrow 0} U(x, t)$ and $U(x, t)=u_{0 y}(E(x-y, t))$. Let $\widetilde{U}(x, t)$ be defined 
by $\widetilde{U}(x, t)=u_{0 y}(E(x-y, t))$. Then for any $t_{0}>0$, if we take $a>0$ sufficiently small and $B>0$ sufficiently large, we have

$$
\begin{aligned}
\int \tilde{U}\left(x, t_{0}\right) \varphi(x) d x & =\int u_{0 y}\left(E\left(x-y, t_{0}\right)\right) \varphi(x) d x \\
& =u_{0 y}\left(\int E\left(x-y, t_{0}\right) \varphi(x) d x\right) \\
& =\int U\left(y, t_{0}\right) \exp \left[-t_{0} \Delta\right]\left(\int E\left(x-y, t_{0}\right) \varphi(x) d x\right) d y \\
& =\int U\left(x, t_{0}\right) \varphi(x) d x, \quad \varphi=\mathcal{S}_{r, a}^{1 / 2, B} .
\end{aligned}
$$

Thus we have $\widetilde{U}(x, t)=U(x, t)$.

Q.E.D.

\section{§3. Ordinary differential equations with constant coefficients}

An ordinary differential operator with constant coefficients is denoted by

$$
p(d / d x)=(d / d x)^{m}+a_{1}(d / d x)^{m-1}+\cdots+a_{m-1}(d / d x)+a_{m}, \quad a_{j} \in \mathfrak{C} .
$$

TheOREM 3.1. Consider the equation

$$
p(d / d x) u(x)=f, \quad f \in\left(\mathcal{S}_{r}^{s}(R)\right)^{\prime} .
$$

For any $f \in\left(\mathcal{S}_{r}^{s}(R)\right)^{\prime}, 1 / 2 \leq r<1,1 / 2 \leq s<\infty$, one can find a solution $u$ in the same space.

Proof. First we shall consider the case where

$$
p(d / d x)=d / d x-A, \quad A \in \mathfrak{C} .
$$

Let $F(x, t)=f_{y}(E(x-y, t))$ then the problem is reduced to seeking a function satisfying the following conditions:

$$
(\partial / \partial x-A) U(x, t)=F(x, t) \text { in } R_{+}^{2},
$$

$$
\left(\partial / \partial t-\partial^{2} / \partial x^{2}\right) U(x, t)=0 \text { in } R_{+}^{2} .
$$

A solution can be obtained in the form

$$
U(x, t)=u_{0}(t) e^{A x}+e^{A x} \int_{0}^{x} e^{-A \xi} F(\xi, t) d \xi, \quad t>0 .
$$


Differentiating both sides, we have

$$
\begin{aligned}
U_{t} & =u_{0}^{\prime}(t) e^{A x}+e^{A x} \int_{0}^{x} e^{-A \xi} F_{t}(\xi, t) d \xi \\
& =e^{A x}\left(u_{0}^{\prime}(t)+\int_{0}^{x} e^{-A \xi} F_{\xi \xi}(\xi, t) d \xi\right) \\
& =U_{x x}=A^{2}\left(u_{0}^{2}(t) e^{A x}+e^{A x} \int_{0}^{x} e^{-A \xi} F(\xi, t) d \xi\right)+A F(x, t)+F_{x}(x, t),
\end{aligned}
$$

from which we have

$$
u_{0}^{\prime}(t)=A^{2} u_{0}(t)+A F(0, t)+F_{x}(0, t), \quad t>0 .
$$

We remark that the similar estimates as $(2.4)$ or $(2.4)^{\prime}$ hold for $F_{x}(x, t)$. Solving the above equation we have

$$
\begin{gathered}
u_{0}(t)=u_{0}(1) e^{A^{2}(t-1)}+e^{A^{2} t} \int_{1}^{t} e^{-A^{2} \tau}\left(A F(0, \tau)+F_{x}(0, \tau)\right) d \tau \\
0<t \leq 1
\end{gathered}
$$

and the estimate of the form

$$
\left|u_{0}(t)\right| \leq C_{\varepsilon} \exp \left[\varepsilon(1 / t)^{1 /(2 s-1)}\right], \quad 0<t \leq 1,(s>1 / 2) .
$$

Combining this with (3.1) we have the estimate when $s>1 / 2$

$$
|U(x, t)| \leq C_{\varepsilon} \exp \left[\varepsilon\left(|x|^{1 / r}+(1 / t)^{1 /(2 s-1)}\right)\right], \quad x \in R, 0<t<1 .
$$

When $s=1 / 2(3.6)$ is replaced by

$$
|U(x, t)| \leq C_{\varepsilon, t} \exp \left[\varepsilon|x|^{1 / r}\right], \quad 0<t<1 .
$$

This means that we have a solution $u=U(x, 0) \in \mathcal{S}_{r}^{s}(R)^{\prime}$ of the equation (3.1) in case the order $m=1$. We can prove the general case by induction on $m$.

\section{§4. Partial differential equations with constant coefficients}

Let us recall that $\mathcal{A}=\mathcal{A}\left(R^{n}\right)$ denotes the set of real analytic functions in $R^{n}$.

Theorem 4.1. Assume that $1 / 2 \leq r, s<\infty$. Then for any $f \in \mathcal{S}_{r}^{s}\left(R^{n}\right)^{\prime}$ there exists a solution $u \in \mathcal{S}_{r}^{s}\left(R^{n}\right)^{\prime}$ to the equation

$$
\Delta u=f \quad \text { in } R^{n} \text {. }
$$


Furthermore if $f \in \mathcal{A}\left(R^{n}\right)$ and satisfies the estimate

$$
|f(x)| \leq C_{\varepsilon} \exp \left[\varepsilon|x|^{1 / r}\right], \quad x \in R^{n}
$$

for any $\varepsilon>0$. Then any solution $u \in \mathcal{S}_{r}^{s}\left(R^{n}\right)^{\prime}$ is also in $\mathcal{A}$.

Proof. Let $f \in \mathcal{S}_{r}^{s}\left(R^{n}\right)$. Then the equation (4.1) is solved by

$$
u(x)=-(2 \pi)^{-n} \int e^{i\langle x, \xi\rangle}|\xi|^{-2} \widetilde{f}(\xi) d \xi
$$

where

$$
\widetilde{f}(\xi)=\int e^{-i\langle x, \xi\rangle} f(x) d x
$$

is the Fourier transform of $f$ and $\xi$-integration in (4.2) is shifted into the complex space at the origin $\xi=0$. We note that we have

$$
\mathcal{S}_{r}^{s}\left(R_{x}^{n}\right)=\mathcal{S}_{s}^{r}\left(R_{\xi}^{n}\right), \quad 0<r, s<\infty, r+s \geq 1 . \quad \text { (c.f. [4].) }
$$

By using the duality we can solve the equation (4.1) in case $f \in \mathcal{S}_{r}^{s}\left(R^{n}\right)^{\prime}$. We denote by $u=\Delta^{-1} f, f \in \mathcal{S}_{r}^{s}$, given in (4.2). Now let $f \in \mathcal{S}_{r}^{s}\left(R^{n}\right)^{\prime}$ then if we define

$$
u(\varphi) \equiv f\left(\Delta^{-1} \varphi\right), \quad \varphi \in \mathcal{S}_{r}^{s}
$$

we have $u \in \mathcal{S}_{r}^{s}\left(R^{n}\right)^{\prime}$ and $u(\Delta \varphi)=f(\varphi)$, for any $\varphi \in \mathcal{S}_{r}^{s}$.

Next let $f \in \mathcal{A}\left(R^{n}\right)$ satisfy the estimate given in the theorem. Let $U(x, t)$ and $F(x, t)$ be the defining functions of $u$ and $f$ respectively, then we have

$$
\Delta U(x, t)=U_{t}(x, t)=F(x, t) \text { in } R_{+}^{n+1},
$$

from where we have

$$
U(x, t)=U(x, 1)+\int_{1}^{t} F(x, \tau) d \tau
$$

Passing $t \longrightarrow 0$, we have $u=U(x, 1)+\int_{0}^{1} F(x, \tau) d \tau \in \mathcal{A}\left(R^{n}\right)$.

Q.E.D.

ThEOREM 4.2. The heat equation

$$
\left(\partial / \partial x_{1}-\left(\partial / \partial x_{2}\right)^{2}\right) u\left(x_{1}, x_{2}\right)=0 \text { in } R^{2}
$$

is not hypoelliptic in the space $\mathcal{S}_{r}^{s}\left(R^{2}\right)^{\prime}, 1<s<2,0<r<1-s / 2$.

Proof. Let $\Gamma=\{(\xi, \sqrt{2 \xi} /(1+i)) ; 0 \leq \xi<\infty\}$ be a curve in $\mathfrak{C}^{2}$. We define $u \in \mathcal{S}_{r}^{s}\left(R^{2}\right)^{\prime}, 1<s<2, s<2(1-r), r>0$, such that 


$$
u(\varphi)=\int_{\Gamma} \tilde{\varphi}(-\zeta) d \xi, \quad \varphi \in \mathcal{S}_{r}^{s}\left(R^{2}\right)
$$

where

$$
\widetilde{\varphi}(\zeta)=\int_{R^{2}} \exp \left[-i\left(x_{1} \zeta_{1}+x_{2} \zeta_{2}\right)\right] \varphi\left(x_{1}, x_{2}\right) d x_{1} d x_{2}
$$

We have $u \in \mathcal{S}_{r}^{s}\left(R^{2}\right)^{\prime}$ by virtue of (4.3) and (1.4) and $u$ is a solution to the equation (4.2) in $R^{2}$ and infinitely differentiable in $R^{2} \cap\left\{x_{2}>0\right\}$. For $x>0$, we have

$$
\begin{aligned}
u(x, \sqrt{x}) & =\int_{0}^{\infty} \exp [i(x \xi+\sqrt{2 x \xi} /(1+i))] d \xi \\
& =x^{-1} \int \exp [i(\xi+\sqrt{2 \xi} /(1+i))] d \xi \\
& =C x^{-1} .
\end{aligned}
$$

This proves that $u \notin C^{\infty}$. We note that the heat equation is hypoelliptic in the space $\mathcal{D}^{\{2\}}\left(R^{2}\right)^{\prime} \supset \mathcal{S}_{r}^{2}\left(R^{2}\right)^{\prime}$.

\section{References}

[1] N. Aronszajn, Traces of analytic solutions of the heat equations, Colloque International C.N.R.S. sur lés équations aux dérivées partielles linéaires, Astérisque, 2-3 (1973), 3568.

[ 2 ] M.S. Baouendi, Solvability of partial differential equations in the traces of analytic solutions of the heat equation, Amer. J. Math., (4) 97 (1973), 983-1005.

[ 3 ] S-Y. Chung and D. Kim, Solvability of Mizohata operators, to appear.

[ 4 ] I.M. Gel'fand and G.E. Shilov, Generalized functions, Vol. 2, Spaces of fundamental and generalized functions, Academic Press, New York and London, 1964.

[ 5 ] L.M. Gel'fand and G.E. Shilov, Generalized functions, Vol. 3, Theory of differential equations, Academic Press, New York and London, 1967.

[ 6 ] L. Hörmander, Linear partial differential operators, Springer, Berlin-Göttingen-Heidelberg, 1963.

[ 7 ] H. Komatsu, Ultradistributions I; Structure theorems and a characterization, J. Fac. Sci. Univ. Tokyo, Sect. IA, 20 (1973), 25-105.

[ 8 ] H. Komatsu, Ultradistributions II; The kernel theorem and ultradistributions with support in a submanifold, J. Fac. Sci. Univ. Tokyo, Sect. IA, 24 (1977), 607-628.

[ 9 ] H. Komatsu, Introduction to the theory of the generalized functions, (in Japanese), Iwanami Shoten, 1978.

[10] T. Matsuzawa, A calculus approach to hyperfunctions I, Nagoya Math. J., 108 (1987), 53-66.

[11] T. Matsuzawa, A calculus approach to hyperfunctions II, Trans. Amer. Math. Soc., (2) 313 (1989), 619-654.

[12] T. Matsuzawa, A calculus approach to hyperfunctions III, Nagoya Math. J., 118 (1990), 133-153. 
[13] T. Matsuzawa, Foundation of a calculus approach to hyperfunctions and applications, to appear in Pitman Math. Res. Notes series.

[14] M. Miyake, Solvability of systems of ordinary differential equations in the space of Aronszajn and the determinant over the Weyl algebra, Nagoya Math. J., 117 (1990), 207-225.

\author{
CHEN DONG \\ Department of Mathematics \\ SCHOOL OF SCIENCE \\ NAGOYA UNIVERSITY, JAPAN \\ Tadato MatsuzaWA \\ DEPARTMENT OF MATHEMATICS \\ MEIJO UNIVERSITY, JAPAN
}

